\title{
MS20-P04 | UNIVERSAL ALGORITHM FOR PREDICTION OF NEW CLEAVAGE PLANES IN SINGLE
}

\section{CRYSTALS}

Vaknin, Uriel (Tel Aviv University, Tel Aviv, ISR); Sherman, Dov (Tel Aviv University, Tel Aviv, ISR); Gorfman, Semën (Tel Aviv University, Tel Aviv, ISR)

Cleavage describes the tendency of crystals to break along specific lattice planes. Acquiring information about cleavage is essential for the investigation of mechanical properties such as fracture energy, plasticity and strength. Although cleavage planes are commonly known for some simple materials (e.g. (110) and (111) for silicon), such information about arbitrary crystals is not available. There are no simple computational methods to predict cleavage planes in single crystals, apart from visual inspection of three-dimensional structures using programs like CrystalMaker or VESTA. Developing such methods may contribute to the understanding of physical and mechanical properties of crystals.

This work aims to develop an algorithm and computer program for automatic inspection of crystal structures and prediction of likely cleavage planes for specific structures. This algorithm enumerates all the possible lattice planes (by listing their Miller indices) and counts intersected atoms / bonds for every plane and its position along the related crystallographic direction. We modelled atoms by thermal ellipsoids / probability density functions (PDFs) derived from their atomic displacement parameters (ADPs), which are commonly available from X-ray diffraction experiment. We modelled bonds by virtual atoms (bond charges) at their anticipated Wyckoff positions and empirical PDFs, accounting for bond strength and extension.

We tested our algorithm on simple inorganic crystal structures such as a-SiO ${ }_{2}, \mathrm{LiNbO}_{3}$ and $\mathrm{CaF}_{2}$. Our algorithm can be used for fast and intuitive prediction of cleavage planes to be further approved by rigorous and time-consuming density functional theory calculations. 